\title{
Action of Hecke operators on products of Igusa theta constants with rational characteristics
}

\author{
by \\ Anatoli N. Andrianov (St. Petersburg) \\ and Fedor A. Andrianov (Los Angeles, CA)
}

Introduction. In 1980 H. Yoshida ([Yo(80), p. 243]) proposed a twodimensional analog of the famous Shimura-Taniyama relation between Hasse zeta functions of elliptic curves over the field of rational numbers and Hecke zeta functions of elliptic modular forms. To confirm the Yoshida conjecture, R. Salvati-Manni and J. Top have considered in [SM-T(93)] a number of products of four Igusa theta constants with rational characteristics which (in part hypothetically) are eigenfunctions of all regular Hecke operators with Andrianov zeta function coinciding (up to a finite number of Euler factors) with the Hasse zeta function of an appropriate two-dimensional Abelian variety.

In this paper we begin to study the transformation properties of products of an even number of Igusa theta constants with rational characteristics considered as Siegel modular forms. More specifically, we investigate their transformations under modular substitutions and under the action of regular Hecke operators. For this we interpret products of theta constants as multiple theta functions of sums of squares and then apply explicit transformation formulas for the action of modular substitutions and regular Hecke operators on theta functions of integral quadratic forms (obtained in $[\operatorname{An}(95)]$ and $[\operatorname{An}(96)]$, respectively). In particular, if the class number of the sum of $2 k$ squares is equal to one, we obtain explicit transformation formulas expressing images of the products of $2 k$ theta constants with rational characteristics under the action of regular Hecke operators in the form of linear combinations of similar products (Theorem 4.1).

2000 Mathematics Subject Classification: Primary 11F27, 11F46, 11F60, 11F66.

Key words and phrases: Igusa theta constants, Hecke operators, Siegel modular forms, theta functions of integral quadratic forms, zeta functions of modular forms.

The first author was supported in part by Russian Fund of Fundamental Research Grant \#02-01-00087. 
In forthcoming works we intend to investigate the question of construction of common eigenfunctions for all regular Hecke operators on spaces spanned by products of theta constants with rational characteristics and, possibly, compute the corresponding zeta functions.

Notation. We reserve the letters $\mathbb{N}, \mathbb{Z}, \mathbb{Q}, \mathbb{R}$, and $\mathbb{C}$ for the set of positive rational integers, the ring of rational integers, and the fields of rational numbers, real numbers, and complex numbers, respectively. $\mathbb{A}_{n}^{m}$ is the set of all $m \times n$-matrices with entries in a set $\mathbb{A}, \mathbb{A}^{n}=\mathbb{A}_{1}^{n}$, and $\mathbb{A}_{n}=\mathbb{A}_{n}^{1}$.

If $M$ is a matrix, ${ }^{t} M$ always denotes the transpose of $M$. If the entries of $M$ belong to $\mathbb{C}$, then $\bar{M}$ is the matrix with complex conjugate entries. For a symmetric matrix $Q$ we write $Q[M]={ }^{t} M Q M$ if the product on the right is defined. $1_{g}$ is the unit $g \times g$-matrix, and

$$
J_{g}=\left(\begin{array}{cc}
0 & 1_{g} \\
-1_{g} & 0
\end{array}\right)
$$

We denote by

$$
\mathbb{E}^{m}=\left\{Q=\left.\left(q_{\alpha \beta}\right) \in \mathbb{Z}_{m}^{m}\right|^{t} Q=Q, q_{11}, q_{22}, \ldots, q_{m m} \in 2 \mathbb{Z}\right\}
$$

the set of all even $m \times m$-matrices, i.e. of matrices of integral quadratic forms in $m$ variables $\mathbf{q}(X)=\frac{1}{2} \cdot{ }^{t} X Q X$, where ${ }^{t} X=\left(x_{1}, \ldots, x_{m}\right)$.

We recall that the level of an invertible matrix $Q \in \mathbb{E}^{m}$ (and of the corresponding form) is the least positive integer $q$ satisfying $q Q^{-1} \in \mathbb{E}^{m}$.

1. Products of theta constants as theta functions for sums of squares. Let $\mathbf{m}=\left(\mathbf{m}^{\prime}, \mathbf{m}^{\prime \prime}\right) \in \mathbb{C}_{2 g}$ with $\mathbf{m}^{\prime}, \mathbf{m}^{\prime \prime} \in \mathbb{C}_{g}$ be a complex $2 g$-row vector, and let $Z$ belong to the upper half-plane of genus $g$ :

$$
\mathbb{H}^{g}=\left\{Z=X+\left.i Y \in \mathbb{C}_{g}^{g}\right|^{t} Z=Z, Y>0\right\} .
$$

The Igusa theta constant with characteristic $\mathbf{m}$ is defined by

$$
\theta_{\mathbf{m}}(Z)=\sum_{\mathbf{n} \in \mathbb{Z}_{g}} \exp \left(\pi i\left\{\left(\mathbf{n}+\mathbf{m}^{\prime}\right) Z \cdot{ }^{t}\left(\mathbf{n}+\mathbf{m}^{\prime}\right)+2\left(\mathbf{n}+\mathbf{m}^{\prime}\right) \cdot{ }^{t} \mathbf{m}^{\prime \prime}\right\}\right) .
$$

If the product $A B$ of two matrices $A$ and $B$ is defined and is a square matrix, then clearly $B A$ is also defined and is a square matrix with the same trace as that of $A B$. It follows that the theta constant can be rewritten in the form

$$
\theta_{\mathbf{m}}(Z)=\sum_{\mathbf{n} \in \mathbb{Z}_{g}} \mathbf{e}\left\{Z \cdot{ }^{t}\left(\mathbf{n}+\mathbf{m}^{\prime}\right)\left(\mathbf{n}+\mathbf{m}^{\prime}\right)+2 \cdot{ }^{t} \mathbf{m}^{\prime \prime}\left(\mathbf{n}+\mathbf{m}^{\prime}\right)\right\},
$$

where for a square matrix $A$ we set

$$
\mathbf{e}\{A\}=\exp (\pi i \cdot \sigma(A)),
$$

and $\sigma(A)$ denotes the trace of $A$. Hence, it is easy to see that the product of $r$ theta constants with characteristics $\mathbf{m}_{1}, \ldots, \mathbf{m}_{r}$ can be written in the 
form

$$
\begin{aligned}
& \prod_{j=1}^{r} \theta_{\mathbf{m}_{j}}(Z) \\
= & \sum_{\mathbf{n}_{1}, \ldots, \mathbf{n}_{r} \in \mathbb{Z}_{g}} \mathbf{e}\left\{\sum_{j=1}^{r}\left(Z \cdot{ }^{t}\left(\mathbf{n}_{j}+\mathbf{m}_{j}^{\prime}\right)\left(\mathbf{n}_{j}+\mathbf{m}_{j}^{\prime}\right)+2 \cdot{ }^{t} \mathbf{m}_{j}^{\prime \prime}\left(\mathbf{n}_{j}+\mathbf{m}_{j}^{\prime}\right)\right)\right\} \\
= & \sum_{N \in \mathbb{Z}_{g}^{r}} \mathbf{e}\left\{Z \cdot{ }^{t}\left(N+M^{\prime}\right)\left(N+M^{\prime}\right)+2 \cdot{ }^{t} M^{\prime \prime}\left(N+M^{\prime}\right)\right\},
\end{aligned}
$$

where we set

$$
N=\left(\begin{array}{c}
\mathbf{n}_{1} \\
\vdots \\
\mathbf{n}_{r}
\end{array}\right), \quad M^{\prime}=\left(\begin{array}{c}
\mathbf{m}_{1}^{\prime} \\
\vdots \\
\mathbf{m}_{r}^{\prime}
\end{array}\right), \quad M^{\prime \prime}=\left(\begin{array}{c}
\mathbf{m}_{1}^{\prime \prime} \\
\vdots \\
\mathbf{m}_{r}^{\prime \prime}
\end{array}\right) .
$$

A product of the form (1.3) will be called a theta product of genus $g$ with characteristic matrix $M=\left(M^{\prime}, M^{\prime \prime}\right)$. The following criterion for the vanishing of theta products is a direct consequence of the Igusa result on vanishing of theta constants $([\operatorname{Ig}(72)$, Theorem 1, p. 174]).

Lemma 1.1. The theta product with characteristic matrix $M=\left(M^{\prime}, M^{\prime \prime}\right)$ $\in \mathbb{C}_{2 g}^{r}$ is identically 0 if and only if there is a row $\mathbf{m}_{j}=\left(\mathbf{m}_{j}^{\prime}, \mathbf{m}_{j}^{\prime \prime}\right)$ of $M$ satisfying

$$
2 \mathbf{m}_{j} \in \mathbb{Z}_{2 g}, \quad 2 \mathbf{m}_{j}^{\prime}{ }^{t} \mathbf{m}_{j}^{\prime \prime} \notin \mathbb{Z} .
$$

The main purpose of this paper is to study the action of Hecke operators on the products of theta constants with rational characteristics. The action of Hecke operators on theta functions of integral positive definite quadratic forms of the form

$$
\Theta(V, Z ; Q)=\sum_{N \in \mathbb{Z}_{g}^{r}} \mathbf{e}\left\{Z \cdot Q\left[N-V_{2}\right]+2 \cdot{ }^{t} V_{1} Q N-{ }^{t} V_{1} Q V_{2}\right\},
$$

where $V=\left(V_{1}, V_{2}\right)$ with $V_{1}, V_{2} \in \mathbb{C}_{g}^{r}$ is the characteristic matrix of the theta function, $Z \in \mathbb{H}^{g}$, and where $Q$ is an even positive definite matrix of order $r$, i.e. the matrix of an integral positive definite quadratic form, was considered in $[\operatorname{An}(96)]$. The series (1.4) converges absolutely and uniformly on compact subsets of the complex space $\mathbb{C}_{2 g}^{r} \times \mathbb{H}^{g}$ and therefore determines a complex-analytic function on that space. The formula (1.3) implies that a product of theta constants can be expressed through the theta function (1.4) of the quadratic form $\mathbf{q}_{r}(X)=x_{1}^{2}+\cdots+x_{r}^{2}$ with the matrix $Q_{r}=2 \cdot 1_{r}$ by the formula 


$$
\begin{aligned}
\mathbf{e}\left\{-{ }^{t} M^{\prime \prime} M^{\prime}\right\} & \prod_{j=1}^{r} \theta_{\mathbf{m}_{j}}(Z) \\
= & \sum_{N \in \mathbb{Z}_{g}^{r}} \mathbf{e}\left\{\frac{1}{2} Z\left(2 \cdot 1_{r}\right)\left[N+M^{\prime}\right]+2 \cdot \cdot^{t}\left(\frac{1}{2} M^{\prime \prime}\right)\left(2 \cdot 1_{r}\right) N\right. \\
& \left.+{ }^{t}\left(\frac{1}{2} M^{\prime \prime}\right)\left(2 \cdot 1_{r}\right) M^{\prime}\right\} \\
= & \Theta\left(\left(\frac{1}{2} M^{\prime \prime},-M^{\prime}\right), \frac{1}{2} Z ; Q_{r}\right),
\end{aligned}
$$

which we shall use in the form

$$
\boldsymbol{\theta}(Z, M)=\prod_{j=1}^{r} \theta_{\mathbf{m}_{j}}(Z)=\delta(M) \cdot \Theta\left(V(M), \frac{1}{2} Z ; Q_{r}\right),
$$

where, for $M=\left(M^{\prime}, M^{\prime \prime}\right)$, we set

$$
\begin{aligned}
\delta(M) & =\mathbf{e}\left\{{ }^{t} M^{\prime \prime} M^{\prime}\right\}, \\
V(M) & =\left(V_{1}(M), V_{2}(M)\right)=\left(\frac{1}{2} M^{\prime \prime},-M^{\prime}\right) \\
& =M\left(\begin{array}{cc}
1_{g} & 0 \\
0 & 2 \cdot 1_{g}
\end{array}\right)^{-1} J_{g} .
\end{aligned}
$$

The following property of the function $\delta(M)$ on rational $M$ is an easy consequence of the definition:

$$
\delta\left(M+M_{1}\right)=\delta(M) \quad \text { if } d M \in \mathbb{Z}_{2 g}^{r} \text { and } M_{1} \in 2 d \mathbb{Z}_{2 g}^{r} \text { with } d \in \mathbb{N} .
$$

2. Modular transformations of theta functions and theta products. Here we shall recall basic transformation formulas for the theta functions (1.4) of integral positive definite quadratic forms and then specialize the formulas to the case of sums of squares.

First of all, note that for any matrix $U \in \mathrm{GL}_{r}(\mathbb{Z})$ we have $U \mathbb{Z}_{g}^{r}=\mathbb{Z}_{g}^{r}$ and so, by replacing $N$ by $U N$ in (1.4), we get the identity

$$
\begin{aligned}
\Theta(V, Z ; Q)= & \sum_{N \in \mathbb{Z}_{g}^{r}} \mathbf{e}\left\{Z \cdot Q[U]\left[N-U^{-1} V_{2}\right]+2 \cdot{ }^{t}\left(U^{-1} V_{1}\right) Q[U] N\right. \\
& \left.-{ }^{t}\left(U^{-1} V_{1}\right) Q[U] U^{-1} V_{2}\right\} \\
= & \Theta\left(U^{-1} V, Z, Q[U]\right) .
\end{aligned}
$$

In the case $Q=Q_{r}$ it implies the formula

$$
\boldsymbol{\theta}(Z, U M)=\boldsymbol{\theta}(Z, M) \quad \text { for every } U \in E_{r},
$$


where

$$
E_{r}=E\left(Q_{r}\right)=\left\{U \in \mathrm{GL}_{r}(\mathbb{Z}) \mid{ }^{t} U U=1_{r}\right\}
$$

is the group of units of $Q_{r}$. In fact, by (1.5) and (2.1), we have

$$
\begin{aligned}
\boldsymbol{\theta}(Z, U M) & =\mathbf{e}\left\{{ }^{t}\left(U M^{\prime \prime}\right) U M^{\prime}\right\} \cdot \Theta\left(V(U M), \frac{1}{2} Z ; Q_{r}\right) \\
& =\mathbf{e}\left\{{ }^{t} M^{\prime \prime} M^{\prime}\right\} \cdot \Theta\left(V(M), \frac{1}{2} Z ; Q_{r}\left[U^{-1}\right]\right)=\boldsymbol{\theta}(Z, M) .
\end{aligned}
$$

Next we mention that the function $\Theta(V, Z ; Q)$ is quasiperiodic with respect to the lattice $\left(Q^{-1} \mathbb{Z}_{g}^{r}, \mathbb{Z}_{g}^{r}\right) \subset \mathbb{C}_{2 g}^{r}$. More exactly, for every $T_{1} \in Q^{-1} \mathbb{Z}_{g}^{r}$ and $T_{2} \in \mathbb{Z}_{g}^{r}$, we have

$$
\begin{aligned}
\Theta\left(\left(V_{1}+T_{1}, V_{2}+\right.\right. & \left.\left.T_{2}\right), Z ; Q\right) \\
= & \sum_{N \in \mathbb{Z}_{g}^{r}} \mathbf{e}\left\{Z \cdot Q\left[N-V_{2}-T_{2}\right]+2 \cdot{ }^{t}\left(V_{1}+T_{1}\right) Q\left(N-T_{2}+T_{2}\right)\right. \\
& \left.-{ }^{t}\left(V_{1}+T_{1}\right) Q\left(V_{2}+T_{2}\right)\right\} \\
= & \mathbf{e}\left\{{ }^{t} V_{1} Q T_{2}-{ }^{t} T_{1} Q V_{2}+{ }^{t} T_{1} Q T_{2}\right\} \cdot \Theta\left(\left(V_{1}, V_{2}\right), Z ; Q\right),
\end{aligned}
$$

since $N^{\prime}=N-T_{2}$ ranges over $\mathbb{Z}_{g}^{r}$ along with $N$, and the matrices ${ }^{t} T_{1} Q N^{\prime}$ are integral for all $N^{\prime} \in \mathbb{Z}_{g}^{r}$. In particular, using the above identity with $Q=Q_{r}$, and (1.7), we conclude that for each integral $r \times 2 g$-matrix $S=\left(S^{\prime}, S^{\prime \prime}\right)$ we have

$$
\begin{aligned}
\boldsymbol{\theta}(Z, M+ & S) \\
= & \mathbf{e}\left\{{ }^{t}\left(M^{\prime \prime}+S^{\prime \prime}\right)\left(M^{\prime}+S^{\prime}\right)\right\} \cdot \Theta\left(V(M)+V(S), \frac{1}{2} Z, Q\right) \\
= & \mathbf{e}\left\{{ }^{t}\left(M^{\prime \prime}+S^{\prime \prime}\right)\left(M^{\prime}+S^{\prime}\right)-{ }^{t} M^{\prime \prime} S^{\prime}+{ }^{t} S^{\prime \prime} M^{\prime}-{ }^{t} S^{\prime \prime} S^{\prime}\right\} \\
& \times \Theta\left(V(M), \frac{1}{2} Z, Q_{r}\right) \\
= & \mathbf{e}\left\{2 \cdot{ }^{t} S^{\prime \prime} M^{\prime}\right\} \cdot \boldsymbol{\theta}(Z, M) .
\end{aligned}
$$

The following formulas for integral symplectic transformations of theta functions with respect to the variable $Z$ are particular cases of those proved in $[\operatorname{An}(95)$, Theorems 3.1 and 4.3].

Let $Q$ be an even positive definite matrix of even order $r=2 k$, and $q$ the level of $Q$. Then the theta function $\Theta(V, Z ; Q)$ of genus $g$ of the matrix $Q$ satisfies the functional equation

$$
\begin{aligned}
\operatorname{det}(C Z+D)^{-k} \cdot \Theta(V,(A Z+B)(C Z & \left.+D)^{-1} ; Q\right) \\
& =\chi_{Q}(\mathcal{M}) \cdot \Theta\left(V \cdot{ }^{t} \mathcal{M}^{-1}, Z ; Q\right)
\end{aligned}
$$


for every matrix $\mathcal{M}=\left(\begin{array}{ll}A & B \\ C & D\end{array}\right)$ in the group

$$
\Gamma_{0}^{g}(q)=\left\{\mathcal{M}=\left(\begin{array}{cc}
A & B \\
C & D
\end{array}\right) \in \mathbb{Z}_{2 g}^{2 g} \mid{ }^{t} \mathcal{M} J_{g} \mathcal{M}=J_{g}, C \equiv 0(\bmod q)\right\}
$$

where $J_{g}$ is the matrix (0.1), with the character $\chi$ of the group defined by

$$
\chi_{Q}\left(\left(\begin{array}{ll}
A & B \\
C & D
\end{array}\right)\right)= \begin{cases}1 & \text { if } q=1 \\
\chi_{Q}(\operatorname{det} D) & \text { if } q>1\end{cases}
$$

where $\chi_{Q}$ is the real Dirichlet character modulo $q$ satisfying $\chi_{Q}(-1)=$ $(-1)^{k}$, and

$$
\chi_{Q}(p)=\left(\frac{(-1)^{k} \operatorname{det} Q}{p}\right) \quad(\text { the Legendre symbol })
$$

for each odd prime $p$ not dividing $q$.

Specialization of these formulas to the case of sums of squares allows us to prove the following lemma.

Lemma 2.1. The product (1.5) of an even number $r=2 k$ of theta constants satisfies the functional equation

$$
\begin{aligned}
\operatorname{det}(C Z+D)^{-k} \cdot \boldsymbol{\theta}((A Z+B) & \left.(C Z+D)^{-1}, M\right) \\
& =\boldsymbol{\chi}_{r}(\mathcal{M}) \delta(M) \overline{\delta(M \mathcal{M})} \cdot \boldsymbol{\theta}(Z, M \mathcal{M})
\end{aligned}
$$

for every matrix $\mathcal{M}=\left(\begin{array}{ll}A & B \\ C & D\end{array}\right)$ in the group

$$
\Gamma_{00}^{g}(2)=\left\{\mathcal{M}=\left(\begin{array}{cc}
A & B \\
C & D
\end{array}\right) \in \Gamma^{g}=\Gamma_{0}^{g}(1) \mid B \equiv C \equiv 0(\bmod 2)\right\},
$$

where

$$
\chi_{r}(\mathcal{M})=\chi_{Q_{r}}(\mathcal{M})=\chi_{4}^{k}(\operatorname{det} D)
$$

with the nontrivial Dirichlet character $\chi_{4}$ modulo 4 , and where $\delta$ is the function (1.6).

Proof. By (1.5) we can write

$$
\begin{aligned}
& \operatorname{det}(C Z+D)^{-k} \cdot \boldsymbol{\theta}\left((A Z+B)(C Z+D)^{-1}, M\right) \\
&= \delta(M) \operatorname{det}\left(2 C\left(\frac{1}{2} Z\right)+D\right)^{-k} \\
& \times \Theta\left(V(M),\left(A\left(\frac{1}{2} Z\right)+\frac{1}{2} B\right)\left(2 C\left(\frac{1}{2} Z\right)+D\right)^{-1} ; Q_{r}\right) .
\end{aligned}
$$

Since, clearly,

$$
\mathcal{M}^{\prime}=\left(\begin{array}{ll}
A^{\prime} & B^{\prime} \\
C^{\prime} & D^{\prime}
\end{array}\right)=\left(\begin{array}{cc}
A & \frac{1}{2} B \\
2 C & D
\end{array}\right)=\omega \mathcal{M} \omega^{-1} \in \Gamma_{0}^{g}(4)
$$


with $\omega=\left(\begin{array}{cc}1_{g} & 0 \\ 0 & 2 \cdot 1_{g}\end{array}\right)$, and the level of $Q_{r}$ is 4 , by the formula (2.5) for the matrix $\mathcal{M}^{\prime}$, the last expression is equal to

$$
\delta(M) \chi_{Q_{r}}\left(\mathcal{M}^{\prime}\right) \cdot \Theta\left(V(M)^{t}\left(\mathcal{M}^{\prime}\right)^{-1}, \frac{1}{2} Z ; Q_{r}\right) .
$$

By (1.7) and ${ }^{t} \mathcal{M}^{\prime} J \mathcal{M}^{\prime}=J$ in the form $J^{t}\left(\mathcal{M}^{\prime}\right)^{-1} J^{-1}=\mathcal{M}^{\prime}$, where $J=J_{g}$ is the matrix (0.1), we have

$$
\begin{aligned}
V(M)^{t}\left(\mathcal{M}^{\prime}\right)^{-1} & =M \omega^{-1} J^{t}\left(\mathcal{M}^{\prime}\right)^{-1} J^{-1} \omega \omega^{-1} J=M \omega^{-1} \mathcal{M}^{\prime} \omega \omega^{-1} J \\
& =V(M \mathcal{M}) .
\end{aligned}
$$

Hence,

$$
\begin{aligned}
\operatorname{det}(C Z+D)^{-k} \cdot \boldsymbol{\theta}\left((A Z+B)(C Z+D)^{-1}, M\right) \\
=\delta(M) \chi_{Q_{r}}(\operatorname{det} D) \overline{\delta(M \mathcal{M})} \delta(M \mathcal{M}) \cdot \Theta\left(V(M \mathcal{M}), \frac{1}{2} Z ; Q_{r}\right),
\end{aligned}
$$

which together with (1.5) for $M \mathcal{M}$ in place of $M$ and with the above formulas for $\chi_{Q_{r}}$ proves the lemma.

Now we recall some definitions. The general real positive symplectic group of genus $g$ consists of all real symplectic matrices of order $2 g$ with positive multipliers:

$$
\mathbb{G}^{g}=\operatorname{GSp}_{g}^{+}(\mathbb{R})=\left\{\left.\mathcal{M} \in \mathbb{R}_{2 g}^{2 g}\right|^{t} \mathcal{M} J_{g} M=\mu(\mathcal{M}) J_{g}, \mu(\mathcal{M})>0\right\},
$$

where $J_{g}$ is the matrix (0.1). It is a real Lie group which acts as a group of analytic automorphisms on the $g(g+1) / 2$-dimensional open complex variety $\mathbb{H}^{g}$ by the rule

$$
\mathbb{G}^{g} \ni \mathcal{M}=\left(\begin{array}{ll}
A & B \\
C & D
\end{array}\right): Z \mapsto \mathcal{M}\langle Z\rangle=(A Z+B)(C Z+D)^{-1} \quad\left(Z \in \mathbb{H}^{g}\right) .
$$

By acting on the upper half-plane $\mathbb{H}^{g}$, the general symplectic group also operates on complex-valued functions $F$ on $\mathbb{H}^{g}$ by Petersson operators of integral weights $k$,

$$
\mathbb{G}^{g} \ni \mathcal{M}=\left(\begin{array}{ll}
A & B \\
C & D
\end{array}\right):\left.F \mapsto F\right|_{k} \mathcal{M}=\operatorname{det}(C Z+D)^{-k} F(\mathcal{M}\langle Z\rangle) .
$$

The Petersson operators satisfy the rules

$$
\left.F\right|_{k} \mathcal{M} \mathcal{M}^{\prime}=\left.\left(\left.F\right|_{k} \mathcal{M}\right)\right|_{k} \mathcal{M}^{\prime} \quad\left(\mathcal{M}, \mathcal{M}^{\prime} \in \mathbb{G}^{g}\right) .
$$

Let $\Omega$ be a subgroup of $\mathbb{G}^{g}$ commensurable with the modular group of genus $g, \Gamma^{g}=\Gamma_{0}^{g}(1), \chi$ a character of $\Omega$, that is, a multiplicative homomorphism of $\Omega$ into nonzero complex numbers with kernel of finite index in $\Omega$, and let $k$ be an integer. A complex-valued function $F$ on $\mathbb{H}$ is called a (Siegel) modular form of weight $k$ and character $\chi$ for the group $\Omega$ if the following conditions are satisfied: 
(i) $F$ is a holomorphic function in $g(g+1) / 2$ complex variables on $\mathbb{H}^{g}$.

(ii) For every matrix $\mathcal{M} \in \Omega$, the function $F$ satisfies the functional equation

$$
\left.F\right|_{k} \mathcal{M}=\chi(\mathcal{M}) F
$$

where $\left.\right|_{k}$ is the Petersson operator of weight $k$.

(iii) If $g=1$, then every function $\left.F\right|_{k} M$ with $M \in \Gamma^{1}$ is bounded on each subset of $\mathbb{H}^{1}$ of the form $\mathbb{H}_{\varepsilon}^{1}=\left\{x+i y \in \mathbb{H}^{1} \mid y \geq \varepsilon\right\}$ with $\varepsilon>0$.

The set $\mathfrak{M}_{k}(\Omega, \boldsymbol{\chi})$ of all modular forms of weight $k$ and character $\boldsymbol{\chi}$ for the group $\Omega$ is a finite-dimensional linear space over $\mathbb{C}$.

THEOREM 2.2. The product of an even number $r=2 k$ of thet a constants with rational characteristic matrix $M \in \frac{1}{d} \mathbb{Z}_{2 g}^{r}$, where $d \in \mathbb{N}$, satisfies the functional equation

$$
\left.\boldsymbol{\theta}(Z, M)\right|_{k} \mathcal{M}=\chi_{M}(\mathcal{M}) \boldsymbol{\theta}(Z, M) \quad \text { for every } \mathcal{M} \in \Gamma^{g}(d) \cap \Gamma_{00}^{g}(2),
$$

where

$$
\Gamma^{g}(d)=\left\{\mathcal{M} \in \mathbb{Z}_{2 g}^{2 g} \mid{ }^{t} \mathcal{M} J_{g} \mathcal{M}=J_{g}, \mathcal{M} \equiv 1_{2 g}(\bmod d)\right\},
$$

is the principal congruence subgroup of level $d$ of the modular group $\Gamma^{g}=$ $\Gamma^{g}(1), \Gamma_{00}^{g}(2)$ is the group (2.8), and where

$$
\chi_{M}(\mathcal{M})=\chi_{r}(\mathcal{M}) \mathbf{e}\left\{S(\mathcal{M}){ }^{t} M M\right\}
$$

with

$$
S(\mathcal{M})=\left(\begin{array}{cc}
B+{ }^{t} B-A^{t} B & { }^{t} D-A^{t} D \\
D-1_{g}-C^{t} B & -C^{t} D
\end{array}\right), \quad \mathcal{M}=\left(\begin{array}{cc}
A & B \\
C & D
\end{array}\right)
$$

the matrix $S(\mathcal{M})$ is symmetric.

If the product $\boldsymbol{\theta}(Z, M)$ is not identically zero, then the function $\chi_{M}$ : $\mathcal{M} \mapsto \chi_{M}(\mathcal{M})$ is a character of the group $\Gamma^{g}(d) \cap \Gamma_{00}^{g}(2)$ coinciding with $\chi_{r}$ on $\Gamma^{g}\left(2 d^{2}\right)$; the theta product is a modular form of weight $k$ and character $\chi_{M}$ for the group $\Gamma^{g}(d) \cap \Gamma_{00}^{g}(2)$.

Proof. Since $\mathcal{M} \in \Gamma_{00}^{g}(2)$, by the definition of the Petersson operators of weight $k$, we can rewrite the functional equation (2.7) in the form

$$
\left.\boldsymbol{\theta}(Z, M)\right|_{k} \mathcal{M}=\chi_{r}(\mathcal{M}) \delta(M) \overline{\delta(M \mathcal{M})} \cdot \boldsymbol{\theta}(Z, M \mathcal{M})
$$

Since $\mathcal{M}=\left(\begin{array}{ll}A & B \\ C & D\end{array}\right) \in \Gamma^{g}(d)$ and the matrix $d M$ is integral, we conclude that so is the matrix

$$
\begin{aligned}
T & =\left(T^{\prime}, T^{\prime \prime}\right)=M \mathcal{M}-M=M\left(\mathcal{M}-1_{2 g}\right)=\left(M^{\prime}, M^{\prime \prime}\right)\left(\begin{array}{cc}
A-1_{g} & B \\
C & D-1_{g}
\end{array}\right) \\
& =\left(M^{\prime}\left(A-1_{g}\right)+M^{\prime \prime} C, M^{\prime} B+M^{\prime \prime}\left(D-1_{g}\right)\right),
\end{aligned}
$$


where $M=\left(M^{\prime}, M^{\prime \prime}\right)$. Therefore, by $(2.4)$, we get

$$
\boldsymbol{\theta}(Z, M \mathcal{M})=\boldsymbol{\theta}(Z, M+T)=\mathbf{e}\left\{2 \cdot{ }^{t} T^{\prime \prime} M^{\prime}\right\} \cdot \boldsymbol{\theta}(Z, M) .
$$

The equation (2.14) follows with

$$
\chi_{M}(\mathcal{M})=\chi_{r}(\mathcal{M}) \delta(M) \overline{\delta(M \mathcal{M})} \mathbf{e}\left\{2 \cdot{ }^{t}\left(M^{\prime} B+M^{\prime \prime}\left(D-1_{g}\right)\right) M^{\prime}\right\} .
$$

In order to prove (2.15), it is sufficient to show that

$$
\delta(M) \overline{\delta(M \mathcal{M})} \mathbf{e}\left\{2 \cdot{ }^{t}\left(M^{\prime} B+M^{\prime \prime}\left(D-1_{g}\right)\right) M^{\prime}\right\}=\mathbf{e}\left\{S(\mathcal{M}){ }^{t} M M\right\}
$$

with $S(\mathcal{M})$ defined by $(2.16)$. Set

$$
\mathcal{M}=1_{2 g}+\left(\begin{array}{ll}
A^{\prime} & B^{\prime} \\
C^{\prime} & D^{\prime}
\end{array}\right)
$$

Then, by (1.6), we have

$$
\begin{aligned}
\delta(M \mathcal{M}) & =\delta\left(\left(M^{\prime}, M^{\prime \prime}\right)\left(\begin{array}{cc}
1_{g}+A^{\prime} & B^{\prime} \\
C^{\prime} & 1_{g}+D^{\prime}
\end{array}\right)\right) \\
& =\mathbf{e}\left\{{ }^{t}\left(M^{\prime \prime}+\left(M^{\prime} B^{\prime}+M^{\prime \prime} D^{\prime}\right)\right)\left(M^{\prime}+\left(M^{\prime} A^{\prime}+M^{\prime \prime} C^{\prime}\right)\right)\right\},
\end{aligned}
$$

whence

$$
\begin{aligned}
& \delta(M) \overline{\delta(M \mathcal{M})} \mathbf{e}\left\{2 \cdot{ }^{t}\left(M^{\prime} B+M^{\prime \prime}\left(D-1_{g}\right)\right) M^{\prime}\right\} \\
&=\mathbf{e}\left\{{ }^{t} M^{\prime \prime} M^{\prime}-{ }^{t}\left(M^{\prime \prime}+\left(M^{\prime} B^{\prime}+M^{\prime \prime} D^{\prime}\right)\right)\left(M^{\prime}+\left(M^{\prime} A^{\prime}+M^{\prime \prime} C^{\prime}\right)\right)\right\} \\
& \times \mathbf{e}\left\{2 \cdot{ }^{t}\left(M^{\prime} B^{\prime}+M^{\prime \prime} D^{\prime}\right) M^{\prime}\right\} \\
&= \mathbf{e}\left\{{ }^{t}\left(M^{\prime} B^{\prime}+M^{\prime \prime} D^{\prime}\right) M^{\prime}-{ }^{t} M^{\prime \prime}\left(M^{\prime} A^{\prime}+M^{\prime \prime} C^{\prime}\right)\right\} \\
& \times \mathbf{e}\left\{-{ }^{t}\left(M^{\prime} B^{\prime}+M^{\prime \prime} D^{\prime}\right)\left(M^{\prime} A^{\prime}+M^{\prime \prime} C^{\prime}\right)\right\} .
\end{aligned}
$$

By standard properties of traces of square matrices, the last expression can be rewritten in the form

$$
\begin{aligned}
\mathbf{e}\left\{{ }^{t} M^{\prime}\left(M^{\prime} B^{\prime}+M^{\prime \prime} D^{\prime}\right)-{ }^{t} M^{\prime \prime}\left(M^{\prime} A^{\prime}+M^{\prime \prime} C^{\prime}\right)\right\} \mathbf{e}\left\{-^{t}\left(M\left(\begin{array}{c}
B^{\prime} \\
D^{\prime}
\end{array}\right)\right) M\left(\begin{array}{l}
A^{\prime} \\
C^{\prime}
\end{array}\right)\right\} \\
=\mathbf{e}\left\{{ }^{t} M M\left(\begin{array}{cc}
B^{\prime} & -A^{\prime} \\
D^{\prime} & -C^{\prime}
\end{array}\right)-\left({ }^{t} B^{\prime},{ }^{t} D^{\prime}\right){ }^{t} M M\left(\begin{array}{c}
A^{\prime} \\
C^{\prime}
\end{array}\right)\right\} \\
=\mathbf{e}\left\{\left(\begin{array}{cc}
B & 1_{g}-A \\
D-1_{g} & -C
\end{array}\right){ }^{t} M M-\left(\begin{array}{c}
A-1_{g} \\
C
\end{array}\right)\left({ }^{t} B,{ }^{t} D-1_{g}\right){ }^{t} M M\right\} \\
=\mathbf{e}\left\{\left(\begin{array}{cc}
B+{ }^{t} B-A^{t} B & { }^{t} D-A{ }^{t} D \\
D-1_{g}-C^{t} B & -C^{t} D
\end{array}\right){ }^{t} M M\right\},
\end{aligned}
$$

which proves $(2.15)-(2.17)$. To prove that the matrix $S(\mathcal{M})$ is symmetric, we note that the $g \times g$ blocks $A, B, C$, and $D$ of every matrix $\mathcal{M}=\left(\begin{array}{ll}A & B \\ C & D\end{array}\right)$ with ${ }^{t} \mathcal{M} J_{g} \mathcal{M}=J_{g}$ satisfy

$$
A^{t} B=B^{t} A, \quad C{ }^{t} D=D^{t} C, \quad A^{t} D-B^{t} C=1_{g}
$$


(see, e.g., $[\operatorname{An}(87)$, p. 6]). It follows that $S(\mathcal{M})$ can be written in the form

$$
S(\mathcal{M})=\left(\begin{array}{cc}
B+{ }^{t} B-A^{t} B & { }^{t} D-A^{t} D \\
D-D^{t} A & -C^{t} D
\end{array}\right)
$$

and is symmetric.

If $\boldsymbol{\theta}(Z, M)$ is not identically zero, it follows from (2.12) and (2.14) that the function $\chi_{M}: \mathcal{M} \mapsto \chi_{M}(\mathcal{M})$ is multiplicative. If $\mathcal{M} \in \Gamma^{g}\left(2 d^{2}\right)$, then $S(\mathcal{M})$ is, clearly, divisible by $2 d^{2}$. Therefore, $S(\mathcal{M})^{t} M M$ is integral and divisible by 2 , and so $\mathbf{e}\left\{S(\mathcal{M})^{t} M M\right\}=1$. The rest for $g>1$ follows from the definition of modular forms; condition (iii) for $g=1$ follows from $[\operatorname{Ig}(72)$, Corollary, p. 176].

3. Action of Hecke operators on theta functions. Here we shall recall basic notions and facts related to Hecke operators and specialize the transformation formulas of general harmonic theta functions under the action of regular Hecke operators obtained in $[\mathrm{An}(96)]$ to the case of theta functions (1.4). For details and proofs see [An(87), Chapters 3 and 4], and $[\operatorname{An}(96), \S 5]$.

Let $\Delta$ be a multiplicative semigroup and $\Omega$ a subgroup of $\Delta$ such that every double coset $\Omega \mathcal{M} \Omega$ of $\Delta$ modulo $\Omega$ is a finite union of left cosets $\Omega \mathcal{M}^{\prime}$. Consider the vector space over a field, say $\mathbb{C}$, consisting of all formal finite linear combinations with coefficients in $\mathbb{C}$ of symbols $(\Omega \mathcal{M})$ with $\mathcal{M} \in \Delta$ which are in one-to-one correspondence with left cosets $\Omega \mathcal{M}$ of the set $\Delta$ modulo $\Omega$. The group $\Omega$ naturally acts on this space by right multiplication defined on the symbols $(\Omega \mathcal{M})$ by

$$
(\Omega \mathcal{M}) \omega=(\Omega \mathcal{M} \omega) \quad(\mathcal{M} \in \Delta, \omega \in \Omega) .
$$

We denote by

$$
\mathcal{H}(\Omega, \Delta)=\operatorname{HS}_{\mathbb{C}}(\Omega, \Delta)
$$

the subspace of all $\Omega$-invariant elements. The multiplication of elements of $\mathcal{H}(\Omega, \Delta)$ given by the formula

$$
\left(\sum_{\alpha} a_{\alpha}\left(\Omega \mathcal{M}_{\alpha}\right)\right)\left(\sum_{\beta} b_{\beta}\left(\Omega \mathcal{N}_{\beta}\right)\right)=\sum_{\alpha, \beta} a_{\alpha} b_{\beta}\left(\Omega \mathcal{M}_{\alpha} \mathcal{N}_{\beta}\right)
$$

does not depend on the choice of the representatives $\mathcal{M}_{\alpha} \in \Omega \mathcal{M}_{\alpha}$ and $\mathcal{N}_{\beta} \in \Omega \mathcal{N}_{\beta}$, and turns $\mathcal{H}(\Omega, \Delta)$ into an associative algebra over $\mathbb{C}$ with the unity element $\left(\Omega 1_{\Omega}\right)$, called the Hecke-Shimura ring or HS-ring of $\Delta$ relative to $\Omega$ (over $\mathbb{C}$ ). The elements

$$
(\mathcal{M})=(\mathcal{M})_{\Omega}=\sum_{M_{i} \in \Omega \backslash \Omega \mathcal{M} \Omega}\left(\Omega \mathcal{M}_{i}\right) \quad(\mathcal{M} \in \Delta)
$$

are in one-to-one correspondence with the double cosets of $\Delta$ modulo $\Omega$, belong to $\mathcal{H}(\Omega, \Delta)$, and form a basis of the ring over $\mathbb{C}$. For brevity, the 
symbols $(\Omega \mathcal{M})$ and $(\mathcal{M})$ will be referred to as left and double classes (of $\Delta$ modulo $\Omega$ ), respectively.

We consider the semigroup

$$
\Sigma^{g}=\mathbb{G}^{g} \cap \mathbb{Z}_{2 g}^{2 g}=\left\{\mathcal{M} \in \mathbb{Z}_{2 g}^{2 g} \mid{ }^{t} \mathcal{M} J_{g} \mathcal{M}=\mu(\mathcal{M}) J_{g}, \mu(\mathcal{M})>0\right\}
$$

and its subsemigroups

$$
\begin{aligned}
\Sigma_{q}^{g} & =\left\{\mathcal{M} \in \Sigma^{g} \mid \operatorname{gcd}(\mu(\mathcal{M}), q)=1\right\}, \\
\Sigma_{0}^{g}(q) & =\left\{\mathcal{M}=\left(\begin{array}{ll}
A & B \\
C & D
\end{array}\right) \in \Sigma_{q}^{g} \mid C \equiv 0(\bmod q)\right\}, \\
\Sigma_{00}^{g}(q) & =\left\{\mathcal{M}=\left(\begin{array}{ll}
A & B \\
C & D
\end{array}\right) \in \Sigma_{q}^{g} \mid B \equiv C \equiv 0(\bmod q)\right\}, \\
\Sigma^{g}(q) & =\left\{\mathcal{M} \in \Sigma_{q}^{g} \mid \mathcal{M} \equiv\left(\begin{array}{cc}
\mu(\mathcal{M}) 1_{g} & 0 \\
0 & 1_{g}
\end{array}\right)(\bmod q)\right\}
\end{aligned}
$$

with $q \in \mathbb{N}$. According to $[\operatorname{An}(87)$, Lemma 3.3.5] and similar facts for the groups $\Gamma_{00}^{g}(q)$, for every $q, q^{\prime} \in \mathbb{N}$ with $q \mid q^{\prime}$ the semigroups satisfy

$$
\begin{gathered}
\Sigma_{0}^{g}(q) \cap \Sigma_{q^{\prime}}^{g}=\Gamma_{0}^{g}(q) \Sigma^{g}\left(q^{\prime}\right)=\Sigma^{g}\left(q^{\prime}\right) \Gamma_{0}^{g}(q), \\
\Sigma_{00}^{g}(q) \cap \Sigma_{q^{\prime}}^{g}=\Gamma_{00}^{g}(q) \Sigma^{g}\left(q^{\prime}\right)=\Sigma^{g}\left(q^{\prime}\right) \Gamma_{00}^{g}(q),
\end{gathered}
$$

where

$$
\Gamma_{00}^{g}(q)=\left\{\mathcal{M}=\left(\begin{array}{cc}
A & B \\
C & D
\end{array}\right) \in \Gamma^{g}=\Gamma_{0}^{g}(1) \mid B \equiv C \equiv 0(\bmod q)\right\} .
$$

If $\Omega$ is a subgroup of finite index of the modular group $\Gamma^{g}$, then the pair $\left(\Omega, \Sigma^{g}\right)$ satisfies the conditions of the definition of HS-rings, as also does each pair $(\Omega, \Delta)$ with $\Omega \subset \Delta \subset \Sigma^{g}$, and we can define the corresponding HeckeShimura rings $\mathcal{H}(\Omega, \Delta)$. We shall say that a group $\Omega$ satisfying $\Gamma^{g}(q) \subset$ $\Omega \subset \Gamma^{g}$ is $q$-symmetric if $\Omega \Sigma^{g}(q)=\Sigma^{g}(q) \Omega$. For such $\Omega$ the HS-ring

$$
\mathcal{H}_{\text {reg }}(\Omega)=\mathcal{H}(\Omega, R(\Omega)) \quad \text { with } \quad R(\Omega)=\Omega \Sigma^{g}(q)=\Sigma^{g}(q) \Omega
$$

is called the regular HS-ring of the group $\Omega$ of level $q$. According to Theorem 3.3.3 of $[\mathrm{An}(87)]$, all regular HS-rings for given genus $g$ and level $q$ are isomorphic to each other. In particular, by (3.3) and (3.4) with $q^{\prime}=q$, the groups $\Gamma_{0}^{g}(q)$ and $\Gamma_{00}^{g}(q)$ are $q$-symmetric, and the regular HS-rings of the groups $\Gamma_{0}^{g}(q), \Gamma_{00}^{g}(q)$, and $\Gamma^{g}(q)$,

$$
\begin{aligned}
\mathcal{H}_{0}^{g}(q) & =\mathcal{H}_{\text {reg }}\left(\Gamma_{0}^{g}(q)\right)=\mathcal{H}\left(\Gamma_{0}^{g}(q), \Sigma_{0}^{g}(q)\right), \\
\mathcal{H}_{00}^{g}(q) & =\mathcal{H}_{\mathrm{reg}}\left(\Gamma_{00}^{g}(q)\right)=\mathcal{H}\left(\Gamma_{00}^{g}(q), \Sigma_{00}^{g}(q)\right), \\
\mathcal{H}^{g}(q) & =\mathcal{H}_{\mathrm{reg}}\left(\Gamma^{g}(q)\right)=\mathcal{H}\left(\Gamma^{g}(q), \Sigma^{g}(q)\right)
\end{aligned}
$$

are naturally isomorphic. For example, the isomorphism of the first and 
third rings can be defined as follows. Let

$$
T^{\prime}=\sum_{\alpha} a_{\alpha}\left(\Gamma_{0}^{g}(q) \mathcal{M}_{\alpha}\right) \in \mathcal{H}_{0}^{g}(q),
$$

where the left classes $\left(\Gamma_{0}^{g}(q) \mathcal{M}_{\alpha}\right)$ with $a_{\alpha} \neq 0$ are distinct. Using (3.3), without loss of generality one may assume that all representatives $\mathcal{M}_{\alpha}$ of the left cosets $\Gamma_{0}^{g}(q) \mathcal{M}_{\alpha}$ belong to $\Sigma^{g}(q)$. Then, obviously, we have

$$
T=\sum_{\alpha} a_{\alpha}\left(\Gamma^{g}(q) \mathcal{M}_{\alpha}\right) \in \mathcal{H}^{g}(q),
$$

and the map $T^{\prime} \mapsto T$ is a homomorphic embedding of the ring $\mathcal{H}_{0}^{g}(q)$ into $\mathcal{H}^{g}(q)$. In fact, it is a ring isomorphism, with inverse

$$
\mathcal{H}^{g}(q) \ni \sum_{\beta} b_{\beta}\left(\Gamma^{g}(q) \mathcal{N}_{\beta}\right) \mapsto \sum_{\beta} b_{\beta}\left(\Gamma_{0}^{g}(q) \mathcal{N}_{\beta}\right) .
$$

Note also that the map

$$
\mathcal{M} \mapsto \mathcal{M}^{\prime}=\omega^{-1} \mathcal{M} \omega \quad \text { with } \quad \omega=\omega^{g}(q)=\left(\begin{array}{cc}
1_{g} & 0 \\
0 & q \cdot 1_{g}
\end{array}\right)
$$

defines an isomorphism of the pair $\Gamma_{0}^{g}\left(q^{2}\right) \subset \Sigma_{0}^{g}\left(q^{2}\right)$ with the pair $\Gamma_{00}^{g}(q) \subset$ $\Sigma_{00}^{g}(q)$, which induces an isomorphism

$$
\boldsymbol{\omega}=\boldsymbol{\omega}^{g}(q): \mathcal{H}_{0}^{g}\left(q^{2}\right) \rightarrow \mathcal{H}_{00}^{g}\left(q^{2}\right) .
$$

The ring isomorphisms allow one to transfer various constructions from one ring to another. For example, the Zharkovskaya homomorphisms $\Psi^{g, n}=$ $\Psi_{k, \chi}^{g, n}: \mathcal{H}_{0}^{g}(q) \rightarrow \mathcal{H}_{0}^{n}(q)$, where $g>n \geq 1, k$ is an integer, and $\chi$ is a Dirichlet character modulo $q$ satisfying $\chi(-1)=(-1)^{k}$, can be naturally carried over to the corresponding HS-rings of principal congruence subgroups, so that we get the homomorphisms

$$
\Psi^{g, n}=\Psi_{k, \chi}^{g, n}: \mathcal{H}^{g}(q) \rightarrow \mathcal{H}^{n}(q) \quad(g>n \geq 1)
$$

satisfying the commutative diagram

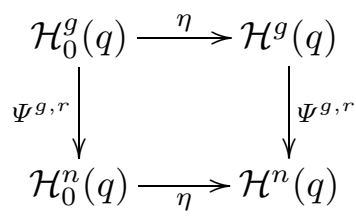

We recall that the Zharkovskaya map from genus $g$ to genus $n$,

$$
\Psi^{g, n}=\Psi_{k, \chi}^{g, n}: \mathcal{H}_{0}^{g}(q) \rightarrow \mathcal{H}_{0}^{n}(q),
$$

can be defined in the following way. Let $T^{\prime}=\sum_{\alpha} a_{\alpha}\left(\Gamma_{0}^{g}(q) \mathcal{M}_{\alpha}\right) \in \mathcal{H}_{0}^{g}(q)$. One can assume that each representative $\mathcal{M}_{\alpha} \in \Gamma_{0}^{g}(q) \backslash \Sigma_{0}^{g}(q)$ is chosen in 
the form

$$
\mathcal{M}_{\alpha}=\left(\begin{array}{cc}
A_{\alpha} & B_{\alpha} \\
0 & D_{\alpha}
\end{array}\right) \quad \text { with } \quad D_{\alpha}=\left(\begin{array}{cc}
D_{\alpha}^{\prime} & * \\
0 & D_{\alpha}^{\prime \prime}
\end{array}\right), D_{\alpha}^{\prime} \in \mathbb{Z}_{n}^{n} .
$$

If $A_{\alpha}=\left(\begin{array}{cc}A_{\alpha}^{\prime} & * \\ * & *\end{array}\right)$ and $B_{\alpha}=\left(\begin{array}{c}B_{\alpha}^{\prime} * \\ *\end{array}\right)$ with $r \times r$-blocks $A_{\alpha}^{\prime}$ and $B_{\alpha}^{\prime}$, then

$$
\mathcal{M}_{\alpha}^{\prime}=\left(\begin{array}{cc}
A_{\alpha}^{\prime} & B_{\alpha}^{\prime} \\
0 & D_{\alpha}^{\prime}
\end{array}\right) \in \Sigma_{0}^{r}(q)
$$

and we put

$$
\Psi_{k, \chi}^{g, n}\left(T^{\prime}\right)=\sum_{\alpha} a_{\alpha}\left|\operatorname{det} D_{\alpha}^{\prime \prime}\right|^{-k} \chi^{-1}\left(\left|\operatorname{det} D_{\alpha}^{\prime \prime}\right|\right)\left(\Gamma_{0}^{n}(q) \mathcal{M}_{\alpha}^{\prime}\right) .
$$

Hecke-Shimura rings act on modular forms and on theta functions by means of a linear representation given by Hecke operators.

First, consider the space $\mathcal{F}=\mathcal{F}(r, g)$ of all real-analytic functions

$$
F=F(V, Z): \mathbb{C}_{2 g}^{r} \times \mathbb{H}^{g} \rightarrow \mathbb{C}
$$

with even $r=2 k$ and define an action of the semigroup $\Sigma_{0}^{g}(q)$, where $q$ is the level of an even positive definite matrix $Q$ of order $r$, on $\mathcal{F}$ by

$$
\begin{aligned}
\Sigma_{0}^{g}(q) \ni \mathcal{M}= & \left(\begin{array}{ll}
A & B \\
C & D
\end{array}\right): \\
& F \mapsto F \mid \mathcal{M}=j(\mathcal{M}, Z)^{-1} F\left(V \cdot{ }^{t} \mathcal{M}, \mathcal{M}\langle Z\rangle\right),
\end{aligned}
$$

where

$$
j(\mathcal{M}, Z)=j_{Q}(\mathcal{M}, Z)=\chi_{Q}(\mathcal{M}) \operatorname{det}(C Z+D)^{k},
$$

$\chi_{Q}$ is the character associated with the matrix $Q$ defined in the previous section, and $\mathcal{M}\langle Z\rangle=(A Z+B)(C Z+D)^{-1}$. It is easy to see that

$$
j\left(\mathcal{M}, \mathcal{M}^{\prime}\langle Z\rangle\right) \cdot j\left(\mathcal{M}^{\prime}, Z\right)=j\left(\mathcal{M M}^{\prime}, Z\right)
$$

for all $\mathcal{M}, \mathcal{M}^{\prime} \in \Sigma_{0}^{g}(q)$ and $Z \in \mathbb{H}^{g}$. Hence,

$$
F|\mathcal{M}| \mathcal{M}^{\prime}=F \mid \mathcal{M} \mathcal{M}^{\prime} \quad\left(F \in \mathcal{F}, \mathcal{M}, \mathcal{M}^{\prime} \in \Sigma_{0}^{g}(q)\right) .
$$

This property of the operators $\mid \mathcal{M}$ allows one to define the standard representation of the Hecke-Shimura ring $\mathcal{H}_{0}^{g}(q)=\mathcal{H}\left(\Gamma_{0}^{g}(q), \Sigma_{0}^{g}(q)\right)$ on the subspace

$$
\mathcal{F}\left(\Gamma_{0}^{g}(q)\right)=\left\{F \in \mathcal{F}|F| \gamma=F, \forall \gamma \in \Gamma_{0}^{g}(q)\right\}
$$

of all $\Gamma_{0}^{g}(q)$-invariant functions of $\mathcal{F}$. Namely, if

$$
T=\sum_{\alpha} a_{\alpha}\left(\Gamma_{0}^{g}(q) \mathcal{M}_{\alpha}\right) \in \mathcal{H}_{0}^{g}(q)
$$

and $F \in \mathcal{F}\left(\Gamma_{0}^{g}(q)\right)$, then the function

$$
F\left|T=\sum_{\alpha} a_{\alpha} F\right| \mathcal{M}_{\alpha}
$$


does not depend on the choice of the representatives $\mathcal{M}_{\alpha} \in \Gamma_{0}^{g}(q) \mathcal{M}_{\alpha}$ and again belongs to $\mathcal{F}\left(\Gamma_{0}^{g}(q)\right)$. The operators $\mid T$ are clearly linear. The map $T \mapsto \mid T$ is linear and, as follows from (2.12) and the definition of multiplication in HS-rings, it satisfies $|T| T^{\prime}=\mid T T^{\prime}$. Thus, we get a linear representation of the ring $\mathcal{H}_{0}^{g}(q)$ on the space $\mathcal{F}\left(\Gamma_{0}^{g}(q)\right)$. The operators $\mid T$ are called the Hecke operators.

By (2.5), the theta function $\Theta(V, Z ; Q)$ with an even positive definite matrix $Q$ of even order $r=2 k$ and level $q$, considered as a function of $V$ and $Z$, belongs to the space $\mathcal{F}\left(\Gamma_{0}^{g}(q)\right)$. By the above, its image

$$
\Theta(V, Z ; Q) \mid T=\sum_{\alpha} a_{\alpha} j\left(\mathcal{M}_{\alpha}, Z\right)^{-1} \Theta\left(V \cdot{ }^{t} \mathcal{M}_{\alpha}, \mathcal{M}_{\alpha}\langle Z\rangle ; Q\right)
$$

under the action of the Hecke operator corresponding to (3.13) does not depend on the choice of the representatives $\mathcal{M}_{\alpha} \in \Gamma_{0}^{g}(q) \mathcal{M}_{\alpha}$ and again belongs to $\mathcal{F}\left(\Gamma_{0}^{g}(q)\right)$. The formulas in $[\operatorname{An}(96)$, Theorem 4.1] express the image of a theta function under the action of Hecke operators as a linear combination with constant coefficients of similar theta functions. In order to formulate the theorem we have to recall two related reductions.

The first reduction relates to each of the HS-rings $\mathcal{H}(\Omega, \Sigma)$ with $\Omega \subset \Gamma^{g}$ and $\Sigma \subset \Sigma^{g}$. By the definition of the semigroup $\Sigma^{g}$, each matrix $M \in \Sigma^{g}$ satisfies ${ }^{t} \mathcal{M} J_{g} \mathcal{M}=\mu(M) J_{g}$, where $\mu(\mathcal{M})$ is a positive integer called the multiplier of $\mathcal{M}$. The multipliers satisfy

$$
\mu\left(\mathcal{M} \mathcal{M}^{\prime}\right)=\mu(\mathcal{M}) \mu\left(\mathcal{M}^{\prime}\right) \quad\left(\mathcal{M}, \mathcal{M}^{\prime} \in \Sigma^{g}\right), \quad \mu(\mathcal{M})=1 \quad \text { if } \mathcal{M} \in \Gamma^{g} .
$$

It follows that $\mu$ takes the same value on each left and each double coset of $\Sigma$ modulo $\Omega$, so one can speak of the multipliers of the cosets. We say that a nonzero formal finite linear combination $T$ of left or double cosets of $\Sigma$ modulo $\Omega$ is homogeneous of multiplier $\mu(T)=\mu$ if all the entering cosets have the same multiplier $\mu$. It is clear that every finite linear combination of cosets is a sum of homogeneous components having different multipliers, and the components are uniquely determined. In particular, this allows one to reduce the consideration of arbitrary Hecke operators $\mid T$ to the case of homogeneous $T$.

Another reduction is related to a special choice of representatives in left cosets $\Omega \mathcal{M}$ when $\Gamma_{0}^{g}(q) \subset \Omega$ and in $\Sigma \subset \Sigma_{0}^{g}(q)$. By Lemma 3.3.4 in $[\operatorname{An}(87)]$, each of the left cosets contains a representative of the form

$$
\begin{aligned}
\mathcal{M} & =\left(\begin{array}{cc}
A & B \\
0 & D
\end{array}\right) \\
& \text { with } \quad A, B, D \in \mathbb{Z}_{g}^{g},{ }^{t} A D=\mu(\mathcal{M}) 1_{g},{ }^{t} B D={ }^{t} D B .
\end{aligned}
$$

Such representatives are often convenient for computations with Hecke operators and will be referred to as triangular representatives. 
The following particular case of Theorem 4.1 in $[\mathrm{An}(96)]$ expresses the images of theta series (1.4) under the action of regular Hecke operators in the form of linear combinations of similar theta series with coefficients explicitly given in terms of certain trigonometric sums.

THEOREM 3.1. Let $Q$ be an even positive definite matrix of even order $r=2 k$ and level $q$. Let

$$
T=\sum_{\alpha} a_{\alpha}\left(\Gamma_{0}^{g}(q) \mathcal{M}_{\alpha}\right)
$$

be a homogeneous element of the Hecke-Shimura ring $\mathcal{H}_{0}^{g}(q)$ with $\mu(T)=\mu$. Assume that for $g<r$ the element $T$ belongs to the image of the ring $\mathcal{H}_{0}^{r}(q)$ under the Zharkovskaya map (3.11) from genus $r$ to genus $g$ with $k=r / 2$ and with character $\chi=\chi_{Q}$ defined in Section 2 ,

$$
T=\Psi_{r / 2, \chi_{Q}}^{r, g}\left(T^{\prime}\right) \quad \text { with } \quad T^{\prime} \in \mathcal{H}_{0}^{r}(q)(g<r) .
$$

Then the image of a theta series (1.5) under the Hecke operator $\mid T$ can be written in the form

$$
\begin{aligned}
& \Theta(V, Z ; Q) \mid T \\
& \quad=\sum_{D \in \Delta(Q, \mu) / \Lambda} I\left(D, Q, \Psi^{g, r}(T)\right) \Theta\left(\mu D^{-1} V, Z ; \mu^{-1} Q[D]\right),
\end{aligned}
$$

where

$$
\begin{aligned}
& \Delta(Q, \mu)=\left\{D \in \mathbb{Z}_{r}^{r} \mid \operatorname{det} D= \pm \mu^{r / 2}, \mu^{-1} Q[D] \in \mathbb{E}^{r}\right\}, \\
& (3.20) \quad \Psi^{g, r}(T)=\Psi_{r / 2, \chi_{Q}}^{g, r}(T)= \begin{cases}\Psi_{r / 2, \chi_{Q}}^{g, r}(T) & \text { if } g>r, \\
T & \text { if } r=g, \\
T^{\prime} \in\left(\Psi_{r / 2, \chi_{Q}}^{r, g}\right)^{-1}(T) & \text { if } g<r,\end{cases}
\end{aligned}
$$

and $I\left(D, Q, T^{\prime}\right)$, for $T^{\prime}$ written in the triangular form

$$
T^{\prime}=\sum_{\beta} b_{\beta}\left(\Gamma_{0}^{r}(q)\left(\begin{array}{cc}
A_{\beta} & B_{\beta} \\
0 & D_{\beta}
\end{array}\right)\right) \in \mathcal{H}_{0}^{r}(q) \quad\left({ }^{t} A_{\beta} D_{\beta}=\mu 1_{r}\right),
$$

are trigonometric sums defined by

$$
=\sum_{\beta ; D^{t} D_{\beta} \equiv 0(\bmod \mu)}^{I\left(D, Q, T^{\prime}\right)} b_{\beta}\left|\operatorname{det} D_{\beta}\right|^{-r / 2} \chi_{Q}^{-1}\left(\left|\operatorname{det} D_{\beta}\right|\right) \mathbf{e}\left\{\mu^{-2} Q[D]^{t} D_{\beta} B_{\beta}\right\},
$$

with $\mathbf{e}\{\ldots\}$ being the exponent (1.2).

As to the condition (3.18), it follows from [An(87), Proposition 5.1.14] that it can be replaced by a more explicit condition of the following lemma.

Lemma 3.2. In the notation of the theorem, a nonzero homogeneous element $T \in \mathcal{H}_{0}^{g}(q)$ with multiplier $\mu(T)=\mu$ belongs to the image of the ring 
$\mathcal{H}_{0}^{r}(q)$ for $r>g$ under the Zharkovskaya map (3.10) from genus $r$ to genus $g$ with $k=r / 2$ and $\chi=\chi_{Q}$ if and only if either $g \geq k$, or $g<k$ and $\chi_{Q}(p)=1$ for each prime $p$ entering the prime factorization of $\mu$ with an odd power.

It is sometimes convenient to rewrite the above transformation formulas in a somewhat different form. In order to do this we have to make several preliminary remarks. We shall say that an even matrix $Q^{\prime}$ is similar to a nonsingular matrix $Q \in \mathbb{E}^{r}, Q^{\prime} \sim Q$, if it can be written in the form $Q^{\prime}=\mu^{-1} Q[D]$ with $D \in \Delta(Q, \mu)$, where $\mu$ is coprime to the level $q$ of $Q$. It easily follows from the definition that the relation of similarity is symmetric. Besides, it is clearly reflexive and transitive. Thus, the set of all nonsingular matrices of $\mathbb{E}^{r}$ is the disjoint union of $s$-classes

$$
s\{Q\}=\left\{Q^{\prime} \in \mathbb{E}^{r} \mid Q^{\prime} \sim Q\right\} \quad\left(Q \in \mathbb{E}^{r}, \operatorname{det} Q \neq 0\right) .
$$

It is easy to see that all matrices of the $s$-class of a matrix $Q$ have the same signature, determinant, level, and divisor as $Q$. Further, we recall that two matrices $Q, Q^{\prime}$ of $\mathbb{E}^{r}$ are said to be equivalent, $Q \simeq Q^{\prime}$, if $Q^{\prime}=Q[U]$ with $U \in \Lambda^{r}=\mathrm{GL}_{r}(\mathbb{Z})$. All matrices equivalent to a matrix $Q$ form the $e$-class of $Q$,

$$
e\{Q\}=\left\{Q^{\prime}=Q[U] \mid U \in \Lambda\right\} .
$$

According to the reduction theory of integral quadratic forms, the $s$-class of every nonsingular matrix $Q \in \mathbb{E}^{r}$ is a finite union of $e$-classes:

$$
s\{Q\}=\bigcup_{j=1}^{h(Q)} e\left\{Q_{j}\right\} .
$$

The quantity $h(Q)$ is called the class number of $Q$.

Now we can reformulate Theorem 3.1 in the following form:

THEOREM 3.3. With the notation and assumptions of Theorem 3.1, the formula (3.19) can be rewritten in the form

$$
\begin{aligned}
& \Theta(V, Z ; Q) \mid T \\
& \quad=\sum_{j=1}^{h^{+}(Q)} \sum_{D \in R\left(Q, \mu Q_{j}\right) / E\left(Q_{j}\right)} I\left(D, Q, \Psi^{g, r}(T)\right) \Theta\left(\mu D^{-1} V, Z, Q_{j}\right),
\end{aligned}
$$

where $Q_{1}, \ldots, Q_{h(Q)}$ is a system of representatives of all different e-classes contained in the s-class of $Q$,

$$
R\left(Q, Q^{\prime}\right)=\left\{M \in \mathbb{Z}_{r}^{r} \mid Q[M]=Q^{\prime}\right\} \quad\left(Q, Q^{\prime} \in \mathbb{E}^{r}\right)
$$

is the set of all integral representations of $Q^{\prime}$ by $Q$, and 


$$
E\left(Q^{\prime}\right)=R\left(Q^{\prime}, Q^{\prime}\right) \quad\left(Q^{\prime} \in \mathbb{E}^{r}, \operatorname{det} Q^{\prime} \neq 0\right)
$$

denotes the group of all units of $Q^{\prime}$.

In particular, if the class number $h(Q)$ of $Q$ is equal to 1 , then (3.22) takes the form

$$
\Theta(V, Z ; Q) \mid T=\sum_{D \in R(Q, \mu Q) / E(Q)} I\left(D, Q, \Psi^{g, r}(T)\right) \Theta\left(\mu D^{-1} V, Z, Q\right) .
$$

Proof. It is an easy consequence of the above definitions that the sums (3.21) are independent of the choice of representatives in the decomposition of $T^{\prime}$ and satisfy

$$
I\left(U D U^{\prime}, Q, T^{\prime}\right)=I\left(D, Q[U], T^{\prime}\right) \quad \text { for all } U, U^{\prime} \in \mathrm{GL}_{m}(\mathbb{Z})
$$

(see [An(96), Lemma 3.1]). From (2.1) and (3.24) we conclude that the term of the sum on the right of (3.19) corresponding to a matrix $D \in$ $\Delta(Q, \mu)$ depends only on the coset $D \Lambda$. On the other hand, each of the matrices $\mu^{-1} Q[D]$ with $D \in \Delta(Q, \mu)$ is similar to $Q$ and so is equivalent to one of the matrices $Q_{1}, \ldots, Q_{h(Q)}$, say, $\mu^{-1} Q[D] \simeq Q_{j}$. This means that $\mu^{-1} Q[D][U]=\mu^{-1} Q[D U]=Q_{j}$ with $U \in \Lambda$. By replacing $D$ by $D U$, we can assume that $D \in R\left(Q, Q_{j}\right)$. If $D^{\prime}=D U^{\prime}$ is another such matrix, then, clearly, $Q_{j}\left[U^{\prime}\right]=Q_{j}$, whence $U^{\prime} \in E\left(Q_{j}\right)$. This proves the formulas.

Note that the sums (3.21) were explicitly computed in $[\mathrm{An}(91)]$ and $[\operatorname{An}(93)]$ for certain generators of the rings $\mathcal{H}_{0}^{r}(q)$ including, in particular, all generators of $\mathcal{H}_{0}^{1}(q)$ and $\mathcal{H}_{0}^{2}(q)$.

4. Action of Hecke operators on theta products. Here we shall consider the action of regular Hecke operators on products of an even number $r=2 k$ of theta constants with rational characteristics considered as modular forms, assuming that the class number $h\left(Q_{r}\right)$ of the sum of $r$ squares is equal to 1 .

Let $F$ belong to the space $\mathfrak{M}_{k}(\Omega)=\mathfrak{M}_{k}(\Omega, \mathbf{1})$ of modular forms of integral weight $k$ and trivial character $\chi=\mathbf{1}$ for a subgroup $\Omega$ of finite index in the modular group $\Gamma^{g}$, and let

$$
T=\sum_{\alpha} a_{\alpha}\left(\Omega \mathcal{M}_{\alpha}\right) \in \mathcal{H}\left(\Omega, \Sigma^{g}\right) .
$$

Then, as easily follows from the definitions of modular forms and HS-rings, and the properties $(2.12),(2.13)$ of the Petersson operators (2.11), the function

$$
F\|T=F\|_{k} T=\left.\sum_{\alpha} a_{\alpha} F\right|_{k} \mathcal{M}_{\alpha}
$$

does not depend on the choice of the representatives $\mathcal{M}_{\alpha} \in \Omega \mathcal{M}_{\alpha}$ and again belongs to the space $\mathfrak{M}_{k}(\Omega)$. These operators are called the Hecke operators 
(of weight $k$ for the group $\Omega$ ). The Hecke operators corresponding to elements of regular HS-rings are called regular. It follows from the definition of multiplication in HS-rings and from (2.12) that the map $T \mapsto \| T$ is a linear representation of the ring $\mathcal{H}(\Omega)$ on the space $\mathfrak{M}_{k}(\Omega)$.

According to Theorem 2.2, if $r=2 k, M \in \frac{1}{d} \mathbb{Z}_{2 g}^{r}$, then the theta product $\boldsymbol{\theta}(Z, M)$ is a modular form of weight $k$ and character $\boldsymbol{\chi}_{r}$ for the group $\Gamma^{g}\left(2 d^{2}\right)$. Assuming in addition that $d$ is even, we have

$$
\boldsymbol{\theta}(Z, M) \in \mathfrak{M}_{k}\left(\Gamma^{g}\left(2 d^{2}\right)\right) .
$$

In this case the action (4.1) of the ring $\mathcal{H}^{g}\left(2 d^{2}\right)$ on the modular form $\boldsymbol{\theta}(Z, M)$ is correctly defined. In order to compute the corresponding images we also assume that $h\left(Q_{r}\right)=1$ and use the formulas (3.23) for the image $\Theta\left(V^{\prime}, Z^{\prime}\right)\left|T^{\prime}=\Theta\left(V^{\prime}, Z^{\prime}, Q_{r}\right)\right| T^{\prime}$, where $V^{\prime}=V(M), Z^{\prime}=\frac{1}{2} Z$, and

$$
T^{\prime}=\sum_{\alpha} a_{\alpha}\left(\Gamma_{0}^{g}(4) \mathcal{M}_{\alpha}^{\prime}\right) \in \mathcal{H}_{0}^{g}(4)
$$

is a homogeneous element of multiplier $\mu\left(T^{\prime}\right)=\mu$ coprime to $d$.

Under the assumptions of Theorem 3.3, by (3.23), we get

$$
\begin{aligned}
\Theta\left(V^{\prime}, Z^{\prime}\right) \mid T^{\prime} & \\
& =\sum_{D \in R\left(Q_{r}, \mu Q_{r}\right) / E\left(Q_{r}\right)} I\left(D, Q_{r}, \Psi^{g, r}\left(T^{\prime}\right)\right) \Theta\left(\mu D^{-1} V^{\prime}, Z^{\prime}\right) .
\end{aligned}
$$

If $\mathcal{M}_{\alpha}^{\prime}=\left(\begin{array}{ll}A_{\alpha}^{\prime} & B_{\alpha}^{\prime} \\ C_{\alpha}^{\prime} & D_{\alpha}^{\prime}\end{array}\right) \in \Sigma_{0}^{g}(4)$, then by (3.12) and (2.15) the left-hand side of (4.4) can be rewritten in the form

$$
\Theta\left(V^{\prime}, Z^{\prime}\right) \mid T^{\prime}=\sum_{\alpha} a_{\alpha} j_{Q_{r}}\left(\mathcal{M}_{\alpha}^{\prime}, Z^{\prime}\right)^{-1} \Theta\left(V^{\prime} \cdot{ }^{t} \mathcal{M}_{\alpha}^{\prime}, \mathcal{M}_{\alpha}^{\prime}\left\langle Z^{\prime}\right\rangle ; Q\right)
$$

Set

$\mathcal{M}_{\alpha}=\left(\begin{array}{cc}A_{\alpha} & B_{\alpha} \\ C_{\alpha} & D_{\alpha}\end{array}\right)=\omega^{-1} \mathcal{M}_{\alpha}^{\prime} \omega=\left(\begin{array}{cc}A_{\alpha}^{\prime} & 2 B_{\alpha}^{\prime} \\ \frac{1}{2} C_{\alpha}^{\prime} & D_{\alpha}^{\prime}\end{array}\right) \in \omega^{-1} \Sigma_{0}^{g}(4) \omega \in \Sigma_{00}^{g}(2)$, where $\omega=\omega^{g}(2)=\left(\begin{array}{cc}1_{g} & 0 \\ 0 & 2 \cdot 1_{g}\end{array}\right)$ (see (3.8)). Then it follows directly from the definitions that

$$
j_{Q_{r}}\left(\mathcal{M}_{\alpha}^{\prime}, Z^{\prime}\right)=\chi_{r}\left(\mathcal{M}_{\alpha}\right) \operatorname{det}\left(C_{\alpha} Z+D_{\alpha}\right)^{k},
$$

where $\chi_{r}$ is the character (2.15), and also

$V^{\prime} \cdot{ }^{t} \mathcal{M}_{\alpha}^{\prime}=V(M) \cdot{ }^{t} \mathcal{M}_{\alpha}^{\prime}=M \omega^{-1} J_{g} \cdot{ }^{t} \mathcal{M}_{\alpha}^{\prime}=M \mu \mathcal{M}_{\alpha}^{-1} \omega^{-1} J_{g}=V\left(M \cdot \mathcal{M}_{\alpha}^{*}\right)$, where $\mathcal{M}_{\alpha}^{*}=\mu \mathcal{M}_{\alpha}^{-1}$, and we have used the relations (1.7) along with the fact that ${ }^{t} \mathcal{M}^{\prime} J_{g} \mathcal{M}^{\prime}=\mu J_{g}$, and finally,

$$
\mathcal{M}_{\alpha}^{\prime}\left\langle Z^{\prime}\right\rangle=\frac{1}{2} \mathcal{M}_{\alpha}\langle Z\rangle
$$


Hence, the left-hand side of (4.4) can be written in the form

$$
\begin{aligned}
& \sum_{\alpha} a_{\alpha} \chi_{r}\left(\mathcal{M}_{\alpha}\right)^{-1} \overline{\delta\left(M \mathcal{M}_{\alpha}^{*}\right)} \operatorname{det}\left(C_{\alpha} Z+D_{\alpha}\right)^{-k} \\
& \times \delta\left(M \mathcal{M}_{\alpha}^{*}\right) \Theta\left(V\left(M \mathcal{M}_{\alpha}^{*}\right), \frac{1}{2} \mathcal{M}_{\alpha}\langle Z\rangle\right) \\
&=\left.\sum_{\alpha} a_{\alpha} \chi_{r}\left(\mathcal{M}_{\alpha}\right)^{-1} \overline{\delta\left(M \mathcal{M}_{\alpha}^{*}\right)} \boldsymbol{\theta}\left(Z, M \mathcal{M}_{\alpha}^{*}\right)\right|_{k} \mathcal{M}_{\alpha},
\end{aligned}
$$

where we have used (1.5) and the definition (2.11) of Petersson operators. On the other hand, since, clearly, $\mu D^{-1} V(M)=V\left(\mu D^{-1} M\right)$, the right-hand side of (4.4) can be rewritten in the form

$\sum_{D \in R\left(Q_{r}, \mu Q_{r}\right) / E\left(Q_{r}\right)} I\left(D, Q_{r}, \Psi^{g, r}\left(T^{\prime}\right)\right) \overline{\delta\left(\mu D^{-1} M\right)} \delta\left(\mu D^{-1} M\right)$

$$
\begin{array}{r}
=\sum_{D \in R\left(Q_{r}, \mu Q_{r}\right) / E\left(Q_{r}\right)} I\left(D, Q_{r}, \Psi^{g, r}\left(T^{\prime}\right)\right) \overline{\delta\left(V\left(\mu D^{-1} M\right), \frac{1}{2} Z\right)} \\
=\sum_{D \in E\left(Q_{r}\right) \backslash R\left(Q_{r}, \mu Q_{r}\right)} I\left(\mu D^{-1}, Q_{r}, \Psi^{g, r}\left(T^{\prime}\right)\right) \overline{\theta(D M)} \boldsymbol{\theta}\left(Z, \mu D^{-1} M\right)
\end{array}
$$

since $D \mapsto \mu D^{-1}$ maps $R\left(Q_{r}, \mu Q_{r}\right)$ into itself and sends right cosets modulo $E\left(Q_{r}\right)$ to left cosets. Hence, (4.4) takes the form

$$
\begin{aligned}
& \left.\sum_{\alpha} a_{\alpha} \chi_{r}\left(\mathcal{M}_{\alpha}\right)^{-1} \overline{\delta\left(M \mathcal{M}_{\alpha}^{*}\right)} \boldsymbol{\theta}\left(Z, M \mathcal{M}_{\alpha}^{*}\right)\right|_{k} \mathcal{M}_{\alpha} \\
& =\sum_{D \in E\left(Q_{r}\right) \backslash R\left(Q_{r}, \mu Q_{r}\right)} I\left(\mu D^{-1}, Q_{r}, \Psi^{g, r}\left(T^{\prime}\right)\right) \overline{\delta(D M)} \boldsymbol{\theta}(Z, D M) .
\end{aligned}
$$

The linear combination

$$
\omega\left(T^{\prime}\right)=\sum_{\alpha} a_{\alpha}\left(\omega^{-1} \Gamma_{0}^{g}(4) \omega \cdot \omega^{-1} \mathcal{M}_{\alpha}^{\prime} \omega\right)=\sum_{\alpha} a_{\alpha}\left(\Gamma_{00}^{g}(2) \mathcal{M}_{\alpha}\right)
$$

belongs to $\mathcal{H}_{00}^{g}(2)$, being the image of $T^{\prime}$ under the map (3.9) for $q=2$. According to (3.4) for $q=2$ and $q^{\prime}=2 d^{2}$, we can assume without loss of generality that all representatives $\mathcal{M}_{\alpha} \in \Gamma_{00}^{g}(2) \mathcal{M}_{\alpha}$ belong to the semigroup $\Sigma^{g}\left(2 d^{2}\right)$. Then the linear combination

$$
T=\sum_{\alpha} a_{\alpha}\left(\Gamma^{g}\left(2 d^{2}\right) \mathcal{M}_{\alpha}\right)
$$

belongs to the ring $\mathcal{H}^{g}\left(2 d^{2}\right)$, it is a homogeneous element of multiplier $\mu(T)=\mu$, and each such element has this form for a homogeneous element $T^{\prime} \in \mathcal{H}_{0}^{g}(4)$ of multiplier $\mu\left(T^{\prime}\right)=\mu$. For $\mu \in \mathbb{N}$ we denote by $[\mu]^{g}$ and 
$[\mu]_{g}$ the $2 g \times 2 g$-matrices

$$
[\mu]^{g}=\left(\begin{array}{cc}
\mu \cdot 1_{g} & 0 \\
0 & 1_{g}
\end{array}\right), \quad[\mu]_{g}=\left(\begin{array}{cc}
1_{g} & 0 \\
0 & \mu \cdot 1_{g}
\end{array}\right) .
$$

Since $\mathcal{M}_{\alpha} \in \Sigma^{g}\left(2 d^{2}\right)$, we have $\mathcal{M}_{\alpha} \equiv[\mu]^{g}\left(\bmod 2 d^{2}\right)$, hence $\mathcal{M}_{\alpha}^{*} \equiv[\mu]_{g}$ $\left(\bmod 2 d^{2}\right)$, that is,

$$
\mathcal{M}_{\alpha}^{*}=[\mu]_{g}+2 d^{2} \mathcal{N} \quad \text { with } \quad \mathcal{N}=\mathcal{N}_{\alpha} \in \mathbb{Z}_{2 g}^{2 g}
$$

(it is easy to see that the map $\mathbb{G}^{g} \ni \mathcal{M} \mapsto \mathcal{M}^{*}=\mu(\mathcal{M}) \mathcal{M}^{-1}$ is an antiautomorphism of the semigroup $\left.\Sigma_{q}^{g}\right)$. It follows that $M \mathcal{M}_{\alpha}^{*}=M[\mu]_{g}+2 d R$ with the integral matrix $R=(d M \mathcal{N})=\left(R^{\prime}, R^{\prime \prime}\right)$. Since the matrix $d M$ is integral, by (1.8) we obtain $\overline{\delta\left(M \mathcal{M}_{\alpha}^{*}\right)}=\overline{\delta\left(M[\mu]_{g}\right)}$ and by (2.4) we have

$$
\boldsymbol{\theta}\left(Z, M \mathcal{M}_{\alpha}^{*}\right)=\mathbf{e}\left(4 d^{t}\left(R^{\prime \prime} M^{\prime}\right)\right) \boldsymbol{\theta}\left(Z, M[\mu]_{g}\right)=\boldsymbol{\theta}\left(Z, M[\mu]_{g}\right) .
$$

Therefore, since clearly $\chi_{r}\left(\mathcal{M}_{\alpha}\right)=1$ for $\mathcal{M}_{\alpha} \in \Sigma^{g}\left(2 d^{2}\right)$ with even $d$, we can rewrite (4.5) in the form

$$
\begin{aligned}
& \overline{\delta\left(M[\mu]_{g}\right)} \boldsymbol{\theta}\left(Z, M[\mu]_{g}\right) \|_{k} T \\
& =\sum_{D \in E\left(Q_{r}\right) \backslash R\left(Q_{r}, \mu Q_{r}\right)} I\left(\mu D^{-1}, Q_{r}, \Psi^{g, r}\left(T^{\prime}\right)\right) \overline{\delta(D M)} \boldsymbol{\theta}(Z, D M) .
\end{aligned}
$$

After these considerations we come to the following theorem.

THeOREM 4.1. Let $r=2 k \in 2 \mathbb{N}$ be such that the class number $h\left(Q_{r}\right)$ of the sum of $r$ squares is equal to $1, g \in \mathbb{N}$, and $M \in \frac{1}{d} \mathbb{Z}_{2 g}^{r}$ with $d \in 2 \mathbb{N}$. Let

$$
T=\sum_{\alpha} a_{\alpha}\left(\Gamma^{g}\left(2 d^{2}\right) \mathcal{M}_{\alpha}\right) \in \mathcal{H}^{g}\left(2 d^{2}\right)
$$

be a homogeneous element with multiplier $\mu(T)=\mu$, such that for the corresponding element

$$
T^{\prime}=\sum_{\alpha} a_{\alpha}\left(\Gamma_{0}^{g}(4)\left(\omega \mathcal{M}_{\alpha} \omega^{-1}\right)\right) \in \mathcal{H}_{0}^{g}(4) \quad \text { with } \quad \omega=\left(\begin{array}{cc}
1_{g} & 0 \\
0 & 2 \cdot 1_{g}
\end{array}\right)
$$

there exists an element of the form $\Psi^{g, r}\left(T^{\prime}\right) \in \mathcal{H}_{0}^{r}(4)$ defined by (3.20). Then the theta product $\boldsymbol{\theta}(Z, M)$ is a modular form of the space $\mathfrak{M}_{k}\left(\Gamma^{g}\left(2 d^{2}\right)\right)$, and its image under the Hecke operator $\|_{k} T$ is again a linear combination of theta products:

$$
\begin{array}{r}
\boldsymbol{\theta}(Z, M) \|_{k} T \\
=\sum_{D \in E\left(Q_{r}\right) \backslash R\left(Q_{r}, \mu Q_{r}\right)} I\left(\mu D^{-1}, Q_{r}, \Psi^{g, r}\left(T^{\prime}\right)\right) \boldsymbol{\theta}\left(Z, D M[\widetilde{\mu}]_{g}\right),
\end{array}
$$

where $I$ are trigonometric sums defined by (3.21), $\widetilde{\mu} \in \mathbb{N}$ is an inverse of $\mu$ modulo $2 d^{2}$, and $[\widetilde{\mu}]_{g}$ is defined by (4.6). 
Proof. The first assertion follows from Theorem 2.2. The formula (4.8) follows from (4.7) if we note that for $D \in R\left(Q_{r}, \mu Q_{r}\right)$,

$$
\delta(D M)=\mathbf{e}\left\{{ }^{t} M^{\prime \prime}{ }^{t} D D M^{\prime}\right\}=\mathbf{e}\left\{\mu^{t} M^{\prime \prime} M\right\}=\delta\left(M[\mu]_{g}\right)
$$

by (1.6), and replace $M$ by $M[\widetilde{\mu}]_{g}$.

As to existence of elements $\Psi^{g, r}\left(T^{\prime}\right)$, specialization of Lemma 3.2 to the case $Q=Q_{r}$ and $\chi_{Q}=\chi_{4}$ gives us the following lemma.

LEMma 4.2. Under the notation of Theorem 4.1, the element $\Psi^{g, r}\left(T^{\prime}\right) \in$ $\mathcal{H}_{0}^{r}(4)$ exists if and only if either $g \geq k$, or $g<k$ and each prime $p$ entering the prime factorization of $\mu=\mu\left(T^{\prime}\right)$ with an odd power satisfies $p \equiv 1$ $(\bmod 4)$.

\section{References}

[An(87)] A. N. Andrianov, Quadratic Forms and Hecke Operators, Grundlehren Math. Wiss. 286, Springer, Berlin, 1987.

[An(91)] - Composition of solutions of quadratic Diophantine equations, Uspekhi Mat. Nauk 46 (1991), no. 2 (278), 3-40; English transl.: Russian Math. Surveys 34 (1991), no. 2, 1-44.

[An(93)] - Factorizations of integral representations of binary quadratic forms, Algebra i Analiz 5 (1993), no. 1, 81-108; English transl.: St. Petersburg Math. J. 5 (1994), no. 1, 71-95.

[An(95)] - Symmetries of harmonic theta-functions of integral quadratic forms, Uspekhi Mat. Nauk 50 (1995), no. 4 (304), 3-44; English transl.: Russian Math. Surveys 50 (1995), no. 4, 661-700.

[An(96)] - Harmonic theta-functions and Hecke operators, Algebra i Analiz 8 (1996), no. 5, 1-31; English transl.: St. Petersburg Math. J. 8 (1997), no. 5, 695-720.

$[\operatorname{Ig}(72)] \quad$ J. I. Igusa, Theta Functions, Grundlehren Math. Wiss. 194, Springer, Berlin, 1972.

[SM-T(93)] R. Salvati-Manni and J. Top, Cusp forms of weight 2 for the group $\Gamma_{2}(4,8)$, Amer. J. Math. 115 (1993), 455-486.

[Yo(80)] H. Yoshida, Siegel's modular forms and the arithmetic of quadratic forms, Invent. Math. 60 (1980), 193-248.

Steklov Mathematical Institute

St. Petersburg Branch

Fontanka 27

191023 St. Petersburg, Russia

E-mail: anandr@pdmi.ras.ru
Department of Mathematics

UCLA

6363 Math. Sciences Building

Box 951555

Los Angeles, CA 90095-1555, U.S.A.

E-mail: fedandr@math.ucla.edu 